\title{
Transitioning ABA Services From in Clinic to Telehealth: Case Study of an Indian Organization's Response to COVID-19 Lockdown
}

\author{
Smita Awasthi ${ }^{1} \cdot$ Sridhar Aravamudhan $^{1}$ (D) $\cdot$ Anupama Jagdish $^{1} \cdot$ Bhavana Joshi $^{1} \cdot$ Papiya Mukherjee $^{1}$.

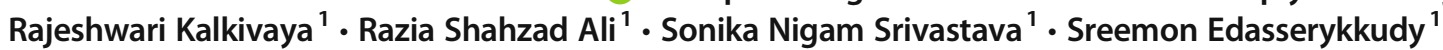

Accepted: 19 April 2021 / Published online: 11 August 2021

(C) Association for Behavior Analysis International 2021

\begin{abstract}
Due to the coronavirus (COVID-19) pandemic, around the middle of March 2020, in-clinic intervention services based in applied behavior analysis provided to children had to be stopped abruptly in India. This qualitative and quantitative case study details how Behavior Momentum India (BMI), an organisation providing ABA-based interventions, transitioned services from in clinic to telehealth while continuing to target each student's skill acquisition goals in language and communication domains. A cohort of 92 students diagnosed with autism or other learning disabilities participated in this study; 51 therapists, 9 behavior supervisors, and a doctoral-level Board Certified Behavior Analyst collaborated with parents; $78 \%$ of the students and $82 \%$ of the therapists used smartphones; and only a few used iPads and laptops. Therapists conducted direct sessions and parent-mediated sessions with 82 students. With 10 students, behavior supervisors trained parents to implement interventions with their children. The critical transition decisions, logistics, and ethical challenges were identified using qualitative methods. Despite significantly reduced session durations, all students continued to acquire targeted skills, and $52 \%$ of the students acquired more skills in telehealth compared to in clinic. A parent satisfaction survey returned high ratings onour organization's initiative, and $72 \%$ of the parents reported that their familiarity and confidence with the science of applied behavior analysis had increased.
\end{abstract}

Keywords Autism $\cdot$ COVID-19 $\cdot$ ABA $\cdot$ Telehealth $\cdot$ Behavioral skills training $\cdot$ Parent training

The coronavirus disease of 2019 (COVID-19), by the end of November 2020, had affected 60,300,000 people worldwide, with a death toll of 1,420,000 (World Health Organization, 2020). In India, in January 2020, the first case of a person who tested positive was registered. Two months later, the government of India ordered a complete nationwide lockdown, restricting the movement of 1,300,000,000 people for 3 weeks initially ("COVID-19 Pandemic Lockdown in India," 2020). Despite a phased unlock process, even after 9 months from the initial lockdown announcement, there was no indication of schools' resumption.

The closure of all educational institutions had an immediate effect on educational, therapeutic, and other intervention services provided to persons with autism and developmental disabilities. Providers of special education and intervention

Smita Awasthi

smita.awasthi@behaviormomentum.com

1 Behavior Momentum India, 407, 7th Main, $80 \mathrm{ft}$. Road, HRBR Layout, Bangalore 560043, India services based in applied behavior analysis (ABA) had to close their clinics with virtually no notice period. Therapists could not travel to their clients' residences to provide services. Families of children with autism and learning disabilities suddenly faced hardships on many fronts. Children with learning disabilities lost their routine of going to special education centers or schools, faced a loss of predictability of their schedules, lost opportunities for social interactions, had extended periods of idleness at home, lost skill acquisition opportunities, and faced probable worsening of inappropriate behaviors. Many parents lost their jobs or had to manage with lower family incomes. They had to divide their attention between their children with and without disability and other extended family members. The lack of availability of domestic help added to parents' workload and strain. For ABA-based intervention service providers, telehealth became an option to examine to maintain continuity of services.

Historically, the delivery of services using telehealth has attracted the attention of researchers and practitioners of behavior analysis primarily to provide services to rural or remote areas with a low concentration of service providers (Wacker et al., 2012, 2013). Lindgren et al. (2016) implemented ABA- 
based telehealth procedures targeting a reduction in problem behaviors. They found that the costs were significantly lower than in-home therapy with telehealth models. Tsami et al. (2019) trained parents to implement functional analysis and functional communication training in eight rural and urban areas in eight countries.

Tomlinson et al. (2018) conducted a systematic review of all peer-reviewed studies that used telehealth to implement behavior-analytic procedures up to July 2017 . Their findings supported telehealth use to train individuals to implement ABA techniques effectively. The training focus in these studies was mostly assessments, such as functional analyses and preference assessments. Some studies addressed intervention strategies for teaching new skills, such as functional communication training (Carr \& Durand, 1985), differential reinforcement interventions, reciprocal imitation training, mand training, and echoic training. However, none of the studies addressed the range of skills typically targeted in intensive behavioral intervention programs in language and communication domains. As the pandemic and the lockdowns continued, practitioners and researchers worldwide showed an increased interest in implementing ABA-based intensive behavioral interventions using telehealth.

During the initial months of the pandemic, several journals, such as Behavior Analysis in Practice, the Journal of Applied Behavior Analysis, the Journal of Behavioural Education, and the Journal of Contextual and Behavioral Science, published articles on special issues addressing the challenges arising out of the pandemic. Frederick et al. (2020), in the United States, implemented a distance support model for 24 students, a majority of whom received services in a general education setting. They targeted the reduction of problem behaviors, task initiation, waiting, reciprocal communication, and social problem solving. In Italy, Espinosa et al. (2020) used parent training to deliver services to 8 minimally verbal students and 16 students they described as verbally interactive. They targeted independent activities, household chores, tabletop activities (emphasizing previously acquired skills), and adult-led or shared activities.

In addition to telehealth sessions with the students, behavioral skills training (BST; Parsons et al., 2012) to train parents on implementing behavioral protocols with fidelity could facilitate the continuation of services during a lockdown. The significant components of training using BST include describing the target skill, providing written or verbal descriptions, demonstrating the skill, having the trainee practice the skill, and providing feedback. Unholz-Bowden et al. (2020) conducted a systematic review of the literature on caregiver training using telehealth. The BST interventions' six most reported components in the studies were performance feedback, within-session instructions, modeling, presession instructions, addressing questions, and written instructions.
With the adoption of any new modality or technology, additional considerations, especially ethical considerations, become important (Peterson et al., 2019; Pollard et al., 2017; Quigley et al., 2019). These included (a) ensuring the compliance of the platform used with regulatory requirements in the region of operation, (b) determining the availability of resources for effective implementation, (c) providing additional oversight of clinical staff, (d) obtaining additional consents for the use of telehealth and recordings, (e) accounting for preexisting relationships in rural communities, (f) accounting for possibilities for dual relationships, (g) maintaining privacy, and (h) considering confidentiality.

Given the extended lockdown for behavioral service providers in countries like India, telehealth adoption for service delivery became important. Further, even if the COVID-19 threat receded, the experience and insights gained from transitioning to telehealth could help provide services to persons with disabilities in rural areas where internet connections may be sufficiently fast and stable.

This qualitative and quantitative study aimed to extend the studies by Espinosa et al. (2020) in Italy and Frederick et al. (2020) in the United States to another country, India. The study also aimed to address additional questions from previous studies, such as the following:

1. Can students with autism spectrum disorder be trained on skills typically addressed in intensive behavioral intervention sessions in clinic (as listed in the Verbal Behavior Milestones Assessment and Placement Program [VBMAPP]; Sundberg, 2008) using telehealth?

2. Can therapists and students use commonly available, lowtech devices such as smartphones for telehealth sessions?

3. What are the various considerations and decisions transitioning ABA-based services to telehealth entails?

4. Can session durations and the rate of trial presentation be maintained at the level of in-clinic sessions?

5. Can students continue to acquire targeted skills? If so, will such acquisition be at a higher rate or a lower rate?

6. Can parent training through telehealth using BST help parents train their children to acquire new skills in the language and communication domains?

\section{Method}

\section{Participants}

The participants in this study were 92 persons with autism and developmental disabilities. They were from Bengaluru, Hyderabad, Mumbai, Noida, and Lucknow in India. They had confirmed diagnoses of autism or other disabilities from a medical professional, such as a developmental pediatrician, 
neurologist, or psychiatrist, or a multidisciplinary team. Students' mean age was 8 years 11 months, with the youngest being 3 years 4 months, and the oldest, 33 years 10 months. Before the lockdown, students received ABA-based behavioral interventions from BMI for periods ranging from 6 months to 7 years, except for one student who enrolled in the intensive behavioral intervention program at the beginning of the lockdown. Their in-clinic sessions daily ranged in duration from 2 to $8 \mathrm{hr}$, barring weekends, public holidays, and periods of illness. Prior to the pandemic, BMI communicated with parents through case meetings, minutes of meetings, and emails. Once the government declared nationwide school closures, BMI sent a communication to parents of all Students receiving services using WhatsApp (WhatsApp LLC, n.d.). In India, there were no regulatory advisories against the use of WhatsApp. BMI used this medium as they felt that parents would attend to messages in WhatsApp earlier and respond to them more quickly. The communication detailed the lockdown implications, the possibility of an extended lockdown, and the proposal to continue providing services using telehealth. It ended by asking parents to respond as a matter of urgency if they would like to continue ABA-based interventions for their children using telehealth and commit to the required time and effort. Parents of 104 students indicated interest and willingness to collaborate, and we initially included them in this study. Twelve students dropped out, attending the sessions for less than 4 weeks, due to constraints at home. They were excluded from the study, taking the number of participants to 92 . The study did not include students who joined telehealth sessions after 2 weeks from the date of commencement, as the objective was to study a cohort of students who started together. Eighty two participants received interventions from therapists and will be referred to as students. Ten participants received interventions from parents and will be referred to as student-children.

\section{Organization and Personnel}

In India, the organization, Behavior Momentum India provided interventions based on ABA to nearly 300 students across six cities through 10 clinics in the prelockdown days. BMI had 230 trained therapists who reported to 9 behavior supervisors: 2 Board Certified Assistant Behavior Analysts and 7 team leaders who had 3-10 years' experience working under the supervision of a Board Certified Behavior Analyst (BCBA). The behavior supervisors received ongoing training and mentoring from the clinical director, the first author of this study and a doctoral-level BCBA.

Fifty female therapists and one male therapist took part in the study. Therapists with less than 1 year of experience under the supervision of a BCBA in conducting behavioral interventions and therapists who were unable to commit time because of their home duties, such as caring for their children and senior members of their extended family, were exempt.

Therapists working in clinics reported to behavior supervisors based in the same clinic. Behavior supervisors assisted the clinical director in formulating and updating the individual education plans of students. They also attended their therapists' intervention sessions, provided feedback, and trained therapists for continuous improvement. The clinical director determined the targeted skills for each student, attended intervention sessions, trained behavior supervisors and therapists, and conducted case meetings with parents along with behavior supervisors. A data administrator collated data received from individual therapists.

\section{Parents and Significant Others}

For 67 students, their mothers played a significant role in facilitating implementation. With the remaining students, their fathers, both parents together, siblings, and caretakers played facilitators' roles (Table 1). Parents had observed their children's behavioral intervention sessions at least once a month in clinic before the lockdown. They had attended case meetings with behavior supervisors and the clinical director at least once in 3 months. Hence all of them were familiar with the skill acquisition targets for their children and the ABA-based protocols used but were not trained in implementing such protocols themselves. During telehealth intervention, parents of 16 students $(17 \%)$ only attended case meetings, parents of 66 students (72\%) facilitated the implementation of sessions by being present throughout the sessions, and parents of 10 students (11\%) implemented interventions with their studentchild directly, with training provided by behavior supervisors. The parent's role is described in more detail in the Procedure section.

\section{Preimplementation Planning, Considerations, and Decisions}

The clinical director, behavior supervisors, therapists, and parents held consultations to arrive at the crucial decisions to transition to telehealth service delivery. The first decision was about technology. In the United States, telehealth systems must be compliant with the Health Insurance Portability

Table 1 Primary Session Facilitators From Families

\begin{tabular}{lll}
\hline Primary collaborator or facilitator & No. of students & $\%$ \\
\hline Mother & 67 & 73 \\
Father & 8 & 9 \\
Both & 12 & 13 \\
Siblings, grandparent, or nanny & 5 & 5 \\
Total & 92 & 100 \\
\hline
\end{tabular}


and Accountability Act (HIPAA, 1996), the Family Educational Rights and Privacy Act (FERPA, 1974), or both. In India, the IT rules under the IT Act of 2000 govern personal data usage and transfer (Vaishnav, 2019). In the event of any negligence in maintaining safety standards while dealing with the data, the rules hold the companies using the data responsible for compensating the affected. The regulations do not proscribe the use of specific video meeting platforms, but there was an advisory against the use of the Zoom app (Ministry of Home Affairs, 2020; "Zoom Video Communications," 2014). BMI decided to use the Meet Now feature of the Skype app (Microsoft, 2020; “Skype," n.d.) or WhatsApp video calling. There were no regulatory advisories against their use in India, and most parents preferred to use these platforms.

The second decision involved the mode of service delivery. If the student was verbally interactive (for a detailed description, see Espinosa et al., 2020) and had a track record of staying on task under instructions for more than $20 \mathrm{~min}$ in in-clinic sessions, BMI decided that a therapist would run the telehealth session directly with the student. This mode would be called the therapist-student direct (TSD) mode. In this mode, a parent only started a session by joining the video meeting and, at the agreed time, ended the session. The therapist would instruct the student to access a tangible reinforcer, such as an iPad, on a thin, variable reinforcement schedule. Another decision was that, with the TSD students, where both the therapist and the student did not have laptops, the skill acquisition targets would not target domains such as mands for tangibles or listener responding, which may require a parent or a caregiver to be present. For other students whose parents consented to telehealth-based behavioral intervention services, with a mutual agreement, BMI decided that at least one parent would be present nearby throughout the session to deliver physical prompts or reinforcers as required by the therapist. This decision especially mitigated a risk of the students engaging in unsafe behaviors in their home environments. This article will refer to this instruction mode as the therapist-student parent-mediated (TSPM) mode. Some parents preferred to receive training from BMI using BST to run instructional sessions with their children. This distance support mode was designated parent behavioral skills training (PBST).BMI decided that only a behavior supervisor would train the parent. A more detailed description of each of these modes is in the Procedure section.

To the extent possible, BMI assigned to each student a therapist who had been implementing behavioral interventions in clinic for at least 3 months with the student. Such an assignment would leverage the rapport the therapist already had with the student, the therapist's knowledge of their student's skill targets, and the implementation protocols. For 11 students in the TSD and TSPM groups, because the therapist working with the student during the previous 3 months was not available, BMI assigned a different therapist with experience with other students with similar skill acquisition targets.

The other decisions involved specific, tangible reinforcers and instructional materials required on the student's end, the initially targeted session durations, and timings based on parent and therapist availability.

\section{Ethical Considerations}

BMI used the messaging and video meeting platforms mentioned previously based on the absence of any adverse advisories from the government, as well as parent preferences. BMI obtained informed consent by email from parents to adopt telehealth and record telehealth sessions periodically for training purposes. The clinical director trained all therapists and behavior supervisors to upload any session they recorded to a secure server residing within the country and delete such recordings from their laptops or phones before 5:30 p.m. each day to prevent any accidental circulation of the recordings. Ethical considerations also included additional training for therapists to implement interventions through telehealth effectively. The study addressed the increased therapist training requirement by behavior supervisors and clinical director overseeing the therapist sessions with students.

\section{Settings and Materials}

TSD students, TSPM students, and parents of PBST students attended telehealth sessions from their homes. This study defines a session as instructional trials and activities conducted for a student after the therapist and student logged in to a WhatsApp video or Skype meeting. All BMI personnel participated from their respective homes. The equipment used included smartphones, tablets, or laptop computers, depending on what was available in each student's home and what was available toBMI personnel. Students on the TSD and TSPM modes received services for one or two sessions a day, from one or two therapists, for 5 to 6 days a week, depending on the timings requested by parents. Therapists using laptops downloaded instructional stimuli, such as pictures of objects or places (e.g., beaches, amusement parks), from the internet from sites offering free reuse images, such as Pixabay. com. They conducted reading and math exercises using stimulus sheets prepared in the student's home based on the therapist's request. Therapists using smartphones sourced the materials for the session from their homes. They displayed the stimuli singly or in an array in front of the smartphone camera. Examples of such materials included everyday objects such as cups, spoons, pencils, glasses, handwritten words on cards, and books. 


\section{Experimental Design}

This study used a case study methodology and qualitative and quantitative approaches. Case studies investigate phenomena occurring in a specific context and use a research strategy covering design, data collection, and data analysis approaches (Gay et al., 2011, pp. 444-445). This study explored, in India, BMI's effort to transition services for a cohort of students from in clinic to telehealth. Qualitative information on the need, the immediate decision challenges, the actions to be taken, and the logistic challenges was enumerated by the clinical director and behavior supervisors during in-depth interviews with the second author, also a BCBA. Quantitative data on the number of students successfully continuing with telehealth services, a comparison of session durations and mastered skills in clinic versus telehealth, and data on the number of trials conducted per student per session at different times during the 6-month intervention were analyzed using a multiple-treatments design. This study does not have a baseline condition but compares two treatment conditions: in clinic and telehealth.

\section{Dependent Variables and Measurement}

\section{TSD and TSPM Sessions}

The primary objectives were to implement the sessions consistently, start with an initial feasible session duration and increase it, conduct maintenance and skill acquisition trials, and get the students to continue acquiring skills. The dependent variables for TSD and TSPM students were daily session duration, the number of instructions per session, and the number of targeted skills acquired.

The therapists recorded the actual duration of sessions for each student in the last 3 days of the 2 nd week of the intervention and the last 3 days of the last week of alternate months after that (Weeks 9, 18, and 26). The therapists uploaded the duration records from the Skype screen or WhatsApp call record screen to a folder in the student's name in a Google Drive shared with the data administrator. The daily session duration was the total of the three durations recorded divided by 3 for each student. Where more than one therapist conducted a session with a student daily, the total of each therapist's session duration was the session duration for the day.

On the 2nd, 9th, 20th, and 28th weeks of the intervention, the therapists recorded the number of trials performed in each of their students' sessions. The mean trials per session for each student were the total number of trials for the week divided by the number of sessions.

The targeted skills and the mastery criteria applicable to each student were available to the behavior supervisors and the therapists. These included targets from the domains listed in the VB-MAPP (Sundberg, 2008), such as mands, tacts, listener responding, visual performance, reading, writing, math, and functional living skills from various domains in Assessment of Functional Living Skills (Partington \& Mueller, 2012). These targets continued to be used in the telehealth sessions with some adaptations, described in the Procedure section. After additional assessments and consultations with parents and the clinical director, the behavior supervisors updated each student's targeted skills every 3 months. The daily first-trial data were taken for each student for each targeted skill by the therapist (Najdowski et al., 2009). On the first trial of the day, the therapist emitted an instruction for each targeted skill, withholding any prompts in the first trial. If the student emitted a correct response in the cold probe for 5 consecutive days, the therapist recorded the skill as acquired. For some students, the number of consecutive days of correct responding in cold probes to meet the mastery criteria was only 3 days. Every Saturday, the therapists sent the list of skills acquired by their students by WhatsApp to the data administrator. The data administrator then entered the data in a Google Sheet shared with their behavior supervisor and the clinical director.

\section{Dependent Variables and Measurement: PBST}

Behavior supervisors trained parents who signed up for the parent training program to implement protocols such as mand training, teaching the student to wait for reinforcement, listener responding, and tacting objects, class, and features using BST. A list of protocols targeted in the first 2 months for the parents in the study is in Table 2 for illustrative purposes. The dependent measures for this intervention were (a) the weekly parent-training duration, (b) the number of protocols parents implemented correctly with their student-child, and (c) the number of targeted skills acquired by the student with the parent implementing the interventions.

Behavior supervisors recorded the duration of their sessions with parents daily for the whole week at the end of Weeks 2, 9, 20, and 28. The duration per week was obtained by totaling the duration of sessions across the week.

The protocols had 5 to 10 steps each. The behavior supervisor measured each protocol's treatment integrity by dividing the number of steps done correctly by the total number of steps. Every Saturday, the parent sent a list of skills acquired by the student to the behavior supervisor.

\section{Interobserver Agreement (IOA)}

\section{IOA on Skills Acquired}

In the TSD and TSPM sessions, the behavior supervisors acted as second observers on one of the two sessions they attended each week with each student. They had a list of skills from the previous weeks reported by the therapist as acquired 
Table 2 List of Implementation Protocols 10 Parents Were Trained In

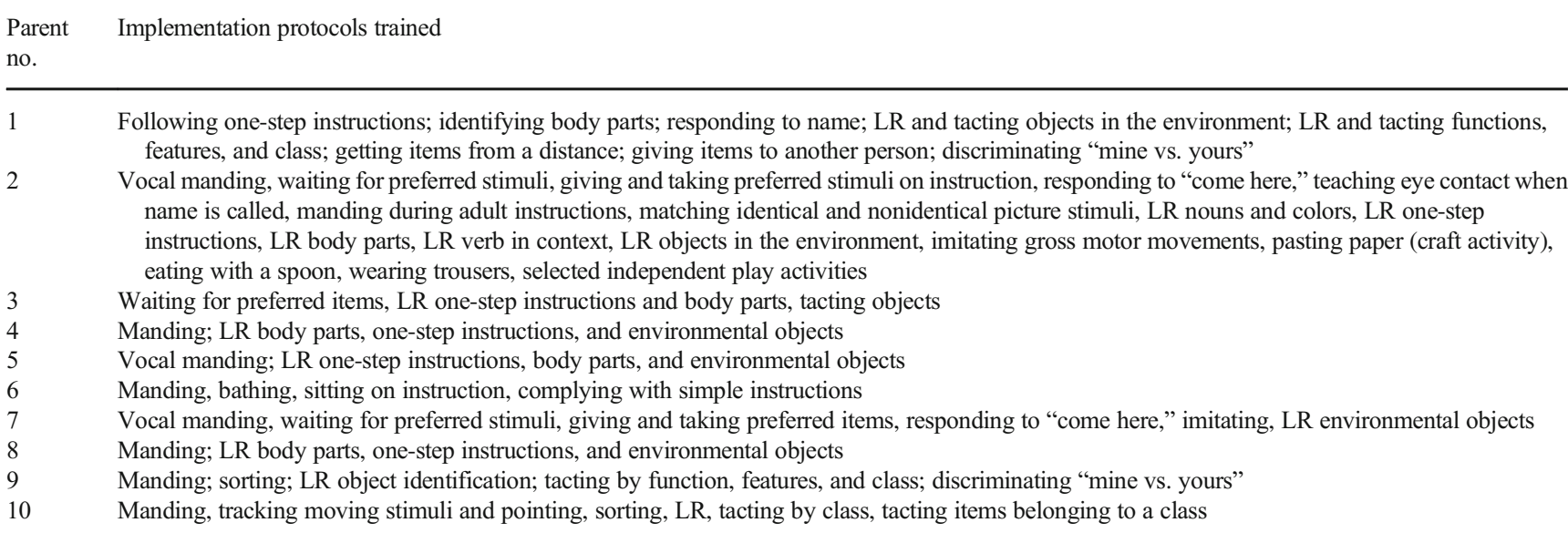

Note. $\mathrm{LR}=$ listener responding.

by their student. During the observation, the second observer recorded a skill as acquired if the student demonstrated that they could perform the skill independently, without any prompts. The behavior supervisor sent data to the data administrator on the skills they confirmed as acquired or not acquired using Google Sheets. The data administrator recorded the data on Google Sheets for the student and the corresponding acquired skills as validated or not validated for each student each month. The interobserver agreement for the number of skills acquired by each student each month was then calculated as the smaller of the number of skills reported as acquired by the therapist and the number of skills reported as acquired by the second observer divided by the larger number reported and multiplied by 100 . For this study, the behavior supervisors validated $82 \%$ of the skills the therapists reported as acquired by their students in this study. The IOA on the skills thus validated was $92 \%$ (range $86 \%-100 \%$ ). The data administrator removed the skills the behavior supervisor reported as "not acquired" from the mastered skills list for any student. The behavior supervisor informed the therapist to resume training on these skills,

\section{IOA on Number of Trials}

A behavior supervisor, acting as a second observer, counted the number of trials on one session of the 2nd, 9th, 20th, and 28th weeks of each participant's sessions. They then compared it with the number of trials reported by the therapist for the same session. The IOA was the smaller of the two numbers divided by the larger, then multiplied by 100 . The mean IOA for this study was $96 \%$ (range $94 \%-100 \%$ ).

\section{IOA on Treatment Integrity of Parent-Delivered Interventions}

The behavior supervisors maintained a list of protocol implementations mastered by parents. The clinical director acted as a second observer for $30 \%$ of these protocols and observed video recordings of parent sessions with their student-child.The clinical director used the same checklist for each protocol as the behavior supervisor to give performance feedback to the parent. The clinical director agreed or disagreed with the behavior supervisor's treatment integrity assessment by scoring using a binary scale of "Yes, I agree" or "No, I disagree." The IOA was the number of protocols where the clinical director scored a "Yes, I agree" divided by the total number of protocol implementations observed by them for each parent. The IOA on this measure for this study was $88 \%$ (range $80 \%-100 \%$ ).

\section{Procedure}

\section{TSD Sessions}

Students assigned to the TSD sessions were verbally interactive. Because parent availability would be minimal, the sessions did not include mand training targets for tangible preferred stimuli that required an adult to be present with the student. The sessions also did not include listenerresponding tasks that required an adult to inform the therapist of selections made by the student. The therapist conducted interventions with the student that involved mands for information; tacts of functions, features, and class; intraverbal responding; reading; math; English grammar; transcription; writing tasks; concept acquisition; and physical exercises.

In the TSD mode, the therapist used only vocal prompts or text prompts. The reinforcement schedules were the same as those used in in-clinic sessions before the lockdown for every student. Therapists with laptops presented stimuli singly or in arrays using PowerPoint slides and shared their screen with the student. They transmitted auditory instructions over the Skype or WhatsApp video platforms. Most therapists used smartphones 
Table 3 Examples of Antecedents and Responses in Telehealth Sessions for Different Types of Trials

\begin{tabular}{|c|c|c|c|}
\hline $\begin{array}{l}\text { Delivery } \\
\text { mode }\end{array}$ & Type of trial & Antecedents, including prompts & Student response \\
\hline \multirow[t]{8}{*}{ TSD } & Tact objects & $\begin{array}{l}\text { The therapist held a spoon in front of the camera and provided } \\
\text { echoic prompts as required. }\end{array}$ & "Spoon" or "This is a spoon." \\
\hline & Tact features & $\begin{array}{l}\text { The therapist held a cup in front of the camera and provided echoic } \\
\text { prompts as required. }\end{array}$ & "This is a cup. It has a rim, a handle, and a base." \\
\hline & Intraverbal & $\begin{array}{l}\text { The therapist asked, "What do you call a large water body } \\
\text { surrounded by land?" and used an echoic prompt, if required. }\end{array}$ & "Lake." \\
\hline & Transcription & The therapist said a word. & $\begin{array}{l}\text { The student wrote the word and held it up in front of the } \\
\text { camera. }\end{array}$ \\
\hline & $\begin{array}{l}\text { Mand for } \\
\text { information } \\
\text { using } \\
\text { "where?" }\end{array}$ & $\begin{array}{l}\text { The therapist said, "Keep the book there," and used a text prompt by } \\
\text { holding up the text in front of the camera or used an echoic } \\
\text { prompt. }\end{array}$ & "Where should I keep the book?" \\
\hline & $\begin{array}{l}\text { Mand for } \\
\text { information } \\
\text { using } \\
\text { "What?" }\end{array}$ & $\begin{array}{l}\text { The therapist said, "Mom is going to give you something special } \\
\text { today," and used a text or echoic prompt. }\end{array}$ & "What is mom going to give me?" \\
\hline & $\begin{array}{l}\text { Mand for } \\
\text { information } \\
\text { using } \\
\text { "Which?" }\end{array}$ & $\begin{array}{l}\text { The student had several colored pens on their table. The therapist } \\
\text { said, "Pick up a pen," and used text or echoic prompts. }\end{array}$ & "Which pen should I pick up?" \\
\hline & $\begin{array}{l}\text { Part listener } \\
\text { responding, } \\
\text { part tact }\end{array}$ & $\begin{array}{l}\text { The therapist presented stimuli in an array on a PowerPoint slide on } \\
\text { their PC or by arranging stimuli in front of their phone camera. } \\
\text { They issued an instruction such as "Which one gives light?" and } \\
\text { used text or echoic prompts. }\end{array}$ & "Candle." \\
\hline \multirow[t]{5}{*}{ TSPM } & $\begin{array}{l}\text { Mand for } \\
\text { tangibles or } \\
\text { edibles }\end{array}$ & $\begin{array}{l}\text { The parent brought an edible or tangible item in view. After } \\
\text { ascertaining interest, the parent provided a model or physical } \\
\text { prompt for the sign or said the name of the item depending on a } \\
\text { predetermined protocol. }\end{array}$ & The student signed or said the name of the item. \\
\hline & $\begin{array}{l}\text { Listener } \\
\text { responding } \\
\text { with arrays }\end{array}$ & $\begin{array}{l}\text { As in TSD, previously, without echoic or text prompts; the therapist } \\
\text { asked which one is a "sea animal." The parent provided a } \\
\text { predetermined gestural prompt if required. }\end{array}$ & $\begin{array}{l}\text { The student touched the image of a ship on their screen. If the } \\
\text { response was correct, the parent gave a thumbs-up. If } \\
\text { incorrect, the parent said the response selected. }\end{array}$ \\
\hline & $\begin{array}{l}\text { Tacts, } \\
\text { intraverbals, } \\
\text { and } \\
\text { transcription }\end{array}$ & As in TSD, previously. & \\
\hline & Reading & $\begin{array}{l}\text { The student was given preprinted worksheets; the therapist had a } \\
\text { copy. }\end{array}$ & The student read words or passages. \\
\hline & Math & $\begin{array}{l}\text { Parent gave a preprinted worksheet with } 100 \text { single-digit addition } \\
\text { exemplars. The therapist had a copy. }\end{array}$ & $\begin{array}{l}\text { The student said or wrote their response and held it up in front } \\
\text { of their camera. }\end{array}$ \\
\hline
\end{tabular}

Note. $\mathrm{TSD}=$ therapist-student direct $\mathrm{TSPM}=$ therapist-student parent mediated.

because they did not have a laptop and thus arranged objects or text stimuli in front of the smartphone camera. For array presentations, the therapist positioned the smartphone with the longer edge resting on the table in the landscape mode, with a support at the back, facing the therapist. They arranged the stimuli in front of the smartphone. The therapist set the smartphone camera to selfie mode. The maximum array size used was four. The student could then see the stimuli, hear the therapist's instructions and prompts, and respond with a vocal-verbal response or a written response as required. The student held up any written response in front of their smartphone camera.

We present some illustrative examples of trials conducted using smartphones in Table 3. In one example, in front of their smartphone camera, the therapist held a cup. They then delivered a discriminative stimulus, such as "Tell me about this." They used echoic prompts as needed to teach the response
"This is a cup. We drink from the cup. It has a handle, a rim, and a base." In another example, the therapist asked a question such as "What do you call a large water body surrounded by land?" The student answered, "It's a lake." In yet another example, the therapist instructed the student to write words with correct spelling, and the student wrote the response in a notebook and held it up for the therapist to see. Additional examples of how trials were conducted to teach manding for information, reading, and math are detailed in Table 3. The students themselves mediated the reinforcer delivery. After a trial or a series of trials, based on the reinforcement schedule, the therapist would ask the student to take a play break; treat themselves to, for example, $40 \mathrm{~s}$ of play with a smartphone; or give themselves a smiley sticker or a token. Once the student, for those on a token economy system, earned a predetermined number of tokens or smileys, the 
therapist told them that they could access a preagreed backup reinforcer.

\section{TSPM Sessions}

These sessions were for students who had difficulty attending to the therapist via video meetings or required physical prompts during training. The targets for skill acquisition, prompting procedures, reinforcement schedules, and mastery criteria were the same for each student as those in clinic. Mand training for tangibles and listener-responding skills continued to be in their education plan. The parent was present, sitting beside the student throughout the session. The prompting method for different types of trials and prompt-fading methods were described to the parent in meetings before the session. The parent delivered preselected reinforcers on a cue from the therapist during the session. The therapist would present an instruction (e.g., "touch your head"), and the parent provided the requisite prompt. In mand-training trials, the therapist asked the parent to bring a preferred stimulus into view. After ascertaining the student's interest, the parent paused for $3 \mathrm{~s}$, provided an echoic prompt or a physical prompt corresponding to the sign, and delivered the preferred stimulus. The therapist paired the word corresponding to the stimulus, such as Play-Doh or chips, with the delivery of the reinforcer. The therapist delivered prompts and faded prompts as required without parent mediation for tact and intraverbal response trials. The therapist positioned their smartphone and stimuli as described in the TSD sessions for listener-responding tasks. After the therapist emitted the discriminative stimulus, such as "Point to the cat," "Point to the animal," or "Touch the sharp one," the parent prompted the student using a gestural or physical prompt. Trials with prompt fading were run by the therapist, who informed the parent just before the trial to use a preagreed reduced prompt level or withdraw the prompt completely. When the student selected a stimulus independently by touching it, it was not visible to the therapist on their screen. Therefore, the parent used a thumbsup sign if the selection was correct and named the stimulus selected if it was incorrect. The therapist then proceeded to conduct the next trial or error correction trials. The therapist also prompted the parent to deliver edibles or access to gadgets or toys at home.

\section{PBST Sessions}

In this mode, behavior supervisors assigned to each parent trained the parent on specific skills to implement behavioral interventions with their student-child. Three behavior supervisors were involved in training a total of 10 parents. All three behavior supervisors had laptops and shared instruction documents and checklists with parents by email. The behavior supervisor and the parent agreed on specific protocols, such as protocols for identifying new reinforcers, mand training, and using discrete-trial training to teach listener-responding skills (Table 2). More than 25 components of BST were described in the literature and used by researchers (Unholz-Bowden et al., 2020, pp. 274-275). The behavior supervisors used presession verbal and written instructions, demonstration, role-play with parents, parents' practicing with their student-child, questionand-answer sessions, and feedback in this study. These components were among the most used components in the previous studies (Unholz-Bowden et al., 2020, p. 276).

The behavior supervisor gave a verbal and written briefing on the steps to be implemented and a checklist for the critical steps to evaluate for treatment integrity. Although the written instructions were detailed, the checklist comprised 5 to 10 critical parent behaviors. The behavior supervisor held a telehealth session in which they explained the implementation steps for each protocol and the treatment integrity checklist items. They listened to and addressed any questions the parent had. They then engaged in a role-play, first with themselves playing the implementer and the parent playing their studentchild's role. They then reversed the roles, with the parent playing the implementer's role and the behavior supervisor playing the student-child's role. The behavior supervisor provided feedback to improve the treatment integrity until all the steps were performed correctly by the parent in the role-play. The parent then practiced the implementation with their student-child and sent recorded videos for each protocol implementation. In the next video meeting, the behavior supervisor provided performance feedback on the number of steps in the checklist executed correctly and incorrectly. If the treatment integrity was more than $80 \%$, the behavior supervisor provided corrective feedback and asked the parent to implement the intervention. If the treatment integrity in any recorded session was less than $80 \%$, the BST for the protocol continued until the treatment integrity in role-plays followed by practice with the student-child improved to $100 \%$. After that, the parent implemented the intervention with their student-child. The behavior supervisor confirmed the student's acquisition of targeted skills based on the video records and planned novel targets in consultation with the parent and the clinical director.

\section{Treatment Integrity}

Behavior supervisors and the clinical director performed treatment integrity checks on the sessions they observed once a month for each therapist-student dyad. They used a checklist of five items to evaluate the session:

1. Did the therapist conduct sufficient trials of previously acquired targets?

2. Did the therapist conduct adequate trials of novel skills targeted for acquisition by the student?

3. Were the reinforcers for the student delivered as per the schedule? 
4. Did the therapist appropriately seek parent facilitation in TSPM sessions?

5. If the student emitted escape behaviors, did the therapist fade the demands and revert to fun activities or to conducting trials on previously mastered skills?

The behavior supervisors or clinical director gave a binary score for the session as "satisfactory" if the therapist scored 4 out of 5 or "needs much improvement" if they scored 3 or less. In the instances where the behavior supervisors or the clinical director rated the session as "needs much improvement," as part of on-the-job training, they provided additional training to the therapist. In this study, there were a total of 312 such observations, of which 287 sessions (92\%) received a satisfactory rating. For PBST sessions, treatment integrity was one of the dependent variables discussed in the sections on response measurement and interobserver agreement.

\section{Social Validity}

Eight weeks into the intervention, a parent satisfaction survey, prepared using Google Forms (Behavior Momentum India, n.d.), was sent to parents of students who received telehealth services. The questions sought information on parents' perception of telehealth implementation and value. The responses to select questions, using the Likert scale provided for each question on the form, are presented in Table 4.

\section{In-Depth Interviews to Identify Logistical Challenges in Implementation}

In this study, from an implementation perspective, it was important to describe the challenges and factors that influenced several critical decisions concerning transitioning to telehealth. Six weeks into the telehealth intervention, a BCBA interviewed the first author, the clinical director, and three behavior supervisors using a questionnaire with a set of open-ended questions. Each interview lasted 30-45 min. The interviewer transcribed their answers to the questions and enumerated the most important decisions, relevant considerations, and challenges to smooth implementation.

\section{Results}

BMI provided services to 16 students with the therapist running sessions directly with the student (TSD), to 66 students with parent mediation (TSPM), and 10 students by training their parents using BST (PBST). Of the 16 TSD students, 14 $(88 \%)$ received services for three or more sessions per week. All the TSPM students received at least five sessions a week. Ten parents received BST over sessions ranging from 1 to 5 days a week, each session lasting 30-60 min. When the government lifted some of the lockdown restrictions in some cities, BMI resumed in-clinic services, on a minimal scale, with several precautions guided by the local medical authorities. Five students from the cohort started attending sessions in clinic again between the $3 \mathrm{rd}$ and 5 th months of the intervention.

\section{Equipment Used}

\section{TSD and TSPM Students}

Of the 82 daily telehealth sessions in the first 2 months, in 64 sessions $(78 \%)$, students had only smartphones, and at the therapist's end, in 67 sessions (82\%), the therapists had only smartphones (Fig. 1). The remaining therapists and students used laptops and, in a few cases, tablets.

\section{PBST Parents and Parent Trainers}

Of the 10 PBST sessions, eight parents used smartphones (80\%), and behavior supervisors used smartphones in $90 \%$ of the sessions (Fig. 2).

\section{Number of Participants and Attrition}

\section{TSD and TSPM Students}

The TSD and TSPM sessions started with a cohort of 82 students; $68(83 \%)$ of the students continued to avail themselves of telehealth ABA-based interventions through the 6 months. A total of 14 TSPM students (17\%) left after the 9th week up to the 28th week (Fig. 3). The top two reasons for leaving telehealth (Fig. 4) were the resumption of sessions in clinic and parents' time constraints.

\section{PBST Parents}

Initially, behavior supervisors trained 10 parents in implementing different protocols based on the education plan drawn up for their student-children. At the end of 2 months, five parents opted out of the BST program. One parent felt they could not learn the protocols and implement them with their student-child, and four others opted out on account of their time constraints.

\section{Session Durations}

\section{TSD and TSPM Students}

The cohort of students received between 2 and $7 \mathrm{hr}$ of behavioral interventions in clinic before the lockdown. The mean daily session duration across 82 TSD and TSPM students, in clinic, was $203 \mathrm{~min}$ (range 60-420). Once the telehealth 
Table 4 Results of Parent Satisfaction Survey From 32 Parents

\begin{tabular}{|c|c|c|c|c|c|c|c|}
\hline \multirow[t]{2}{*}{ No. } & \multirow[t]{2}{*}{ Question } & \multicolumn{6}{|l|}{ Results } \\
\hline & & Scale & Excellent & $\begin{array}{l}\text { Very } \\
\text { satisfactory }\end{array}$ & Satisfactory & Okay & Not satisfactory \\
\hline \multirow[t]{2}{*}{1} & \multirow[t]{2}{*}{$\begin{array}{l}\text { Rate Behavior Momentum India's initiative in } \\
\text { providing telehealth services. }\end{array}$} & $\begin{array}{l}\text { No. of } \\
\quad \text { respondents }\end{array}$ & 16 & 11 & 5 & 0 & 0 \\
\hline & & $\%$ & 50 & 34 & 16 & 0 & 0 \\
\hline \multirow[t]{3}{*}{2} & \multirow[t]{3}{*}{ Rate the therapist's enthusiasm and efforts. } & Scale & Excellent & $\begin{array}{l}\text { Very } \\
\quad \text { satisfactory }\end{array}$ & Satisfactory & Okay & Not satisfactory \\
\hline & & $\begin{array}{l}\text { No. of } \\
\quad \text { respondents }\end{array}$ & 18 & 14 & 0 & 0 & 0 \\
\hline & & $\%$ & 56 & 44 & 0 & 0 & 0 \\
\hline \multirow[t]{3}{*}{3} & \multirow[t]{3}{*}{$\begin{array}{l}\text { Rate the clinical director's recommendations for } \\
\text { your child. }\end{array}$} & Scale used & $\begin{array}{l}\text { Extremely } \\
\quad \text { valuab- } \\
\text { le }\end{array}$ & Very valuable & Valuable & Just okay & Not satisfactory \\
\hline & & $\begin{array}{l}\text { No. of } \\
\quad \text { respondents }\end{array}$ & 17 & 9 & 6 & 0 & 0 \\
\hline & & $\%$ & 53 & 28 & 19 & 0 & 0 \\
\hline \multirow[t]{3}{*}{4} & \multirow[t]{3}{*}{ In this difficult time, my child has acquired ... } & Scale & $\begin{array}{l}\text { Many } \\
\text { targets }\end{array}$ & Few targets & None & No response & \\
\hline & & $\begin{array}{l}\text { No. of } \\
\quad \text { respondents }\end{array}$ & 10 & 21 & 0 & 1 & \\
\hline & & $\%$ & 31 & 66 & 0 & 3 & \\
\hline \multirow[t]{2}{*}{5} & \multirow[t]{2}{*}{$\begin{array}{l}\text { Has telehealth made a difference in your child's } \\
\text { productivity at home? }\end{array}$} & $\begin{array}{l}\text { Scale } \\
\text { No. of } \\
\text { respondents }\end{array}$ & $\begin{array}{l}\text { Yes, a lot } \\
16\end{array}$ & $\begin{array}{l}\text { Yes, a little bit } \\
12\end{array}$ & $\begin{array}{l}\text { Not really } \\
4\end{array}$ & $\begin{array}{l}\text { Not at all } \\
0\end{array}$ & \\
\hline & & $\%$ & 50 & 37 & 1 & 0 & \\
\hline \multirow[t]{3}{*}{6} & \multirow[t]{3}{*}{$\begin{array}{l}\text { Since lockdown, my awareness, familiarity, and } \\
\text { confidence with applied behavior analysis has . }\end{array}$} & Scale & Increased & $\begin{array}{l}\text { Remained the } \\
\text { same }\end{array}$ & Decreased & & \\
\hline & & $\begin{array}{l}\text { No. of } \\
\text { respondents }\end{array}$ & 23 & 8 & 1 & & \\
\hline & & $\%$ & 72 & 25 & 3 & & \\
\hline \multirow[t]{3}{*}{7} & \multirow{3}{*}{$\begin{array}{l}\text { Videos and information shared with Behavior } \\
\text { Momentum India is safe and confidential. }\end{array}$} & Scale & Yes & No & & & \\
\hline & & $\begin{array}{l}\text { No. of } \\
\text { respondents }\end{array}$ & 32 & 0 & & & \\
\hline & & $\%$ & 100 & 0 & & & \\
\hline
\end{tabular}

interventions commenced, the mean session duration measured in Weeks 2, 9, 18, and 26, as well as these durations as a percentage of in-clinic session durations, are presented in Fig. 5. In the telehealth phase, the mean session duration was 36 min (range 15-90) in the 2nd week, and it increased to 72 min (range $30-180$ ) by the 28 th week.

\section{PBST Students: Parent Sessions and Duration}

Initially, 10 parents in the program attended BST training sessions with behavior supervisors for a mean duration of 93 min per week (range 30-300), as shown in Fig. 6. Toward the end of the study, the five parents who had continued attended training sessions for a mean duration of $144 \mathrm{~min}$ per week (range 60-300).

\section{Mean Trials per Hour: TSD and TSPM Students}

The mean number of trials per hour in the 3rd, 9th, 20th, and 28th weeks of the telehealth intervention is in Fig. 7. The mean number of trials per hour increased from 39 (range 1-
180 ) in the 2nd week to 97 (range 36-230) at the end of the study. The corresponding figures for in-clinic sessions are not available.

\section{Number of Skills Acquired}

\section{TSD and TSPM Students}

Figure 8 shows the number of skills the students mastered in 2 months in the prelockdown or in-clinic phase (mid-January 2020 to mid-March 2020) and the telehealth sessions during three successive blocks of 2 months each. The mean number of mastered skills in the in-clinic phase for this cohort of 82 students was 23 (range 0-95). The acquisition rate was comparable in the subsequent blocks of 2 months, reaching 26 (range 1-112) at the end of the study.

The number of skills acquired by each student in the last 2 months of the intervention and the 2 months in clinic before the telehealth intervention is presented in a scatterplot (Fig. 9). An equal number of students gained more skills and fewer 
Fig. 1 Equipment Used at Students' End and Therapists' End for Therapist-Student Direct and Therapist-Student ParentMediated Sessions

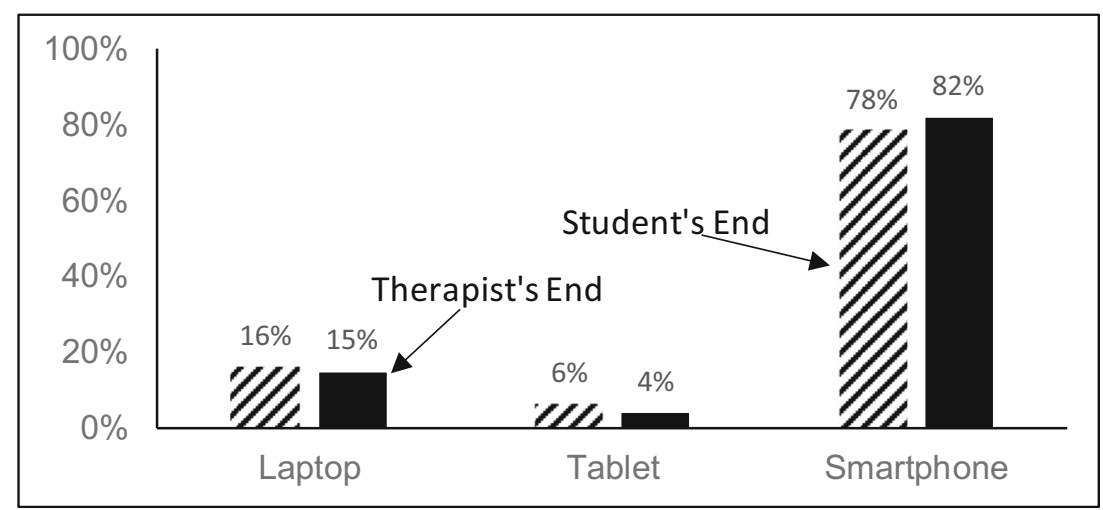

skills than the in-clinic condition, and a few outliers acquired a greater number of skills or much fewer skills.

The number of students in each block of 2 months and the number of students for whom the number of skills acquired increased, maintained, or decreased compared to the in-clinic phase are in Table 5. Compared to the number of skills acquired in clinic, in the first 2 months of the telehealth intervention, the number of skills acquired increased for $46 \%$ of the TSD and TSPM students. In the last 2 months of the study, the number of skills acquired increased for $52 \%$. To determine if a reduction in session duration alone could account for students' acquisition of fewer skills, we calculated the hours of instruction per skill mastered for each student. The data on the mean hours of instruction per mastered skill per student are in Figs. 10 and 11 for the students who acquired fewer and more skills, respectively, in the last 2 months compared to the inclinic condition. For the students who acquired fewer skills in the final 2 months of telehealth compared to the in-clinic condition, there was nearly a fivefold increase in the hours of instruction per mastered skill in the first 2 months, from a mean of 1.4 in the in-clinic condition to 6.7 in the first 2 months of telehealth (Fig. 10). These hours of instruction per skill acquired reduced starting from the $3 \mathrm{rd}$ month. At $3.5 \mathrm{hr}$ of instruction per mastered skill per hour at the end of the study, it was still 2.5 times the hours required in clinic. For the students who acquired more skills in the last 2 months (Fig. 11), the mean hours of instruction required per skill acquisition reduced from $4.5 \mathrm{hr}$ in clinic to $3.0 \mathrm{hr}$ in the last 2 months.

\section{Protocols Mastered by PBST Parents}

The number of protocols each parent mastered across blocks of 2 months from the start of the intervention is in Fig. 12. In the 6-month study period, two parents mastered 29 and 24 protocols, respectively, and seven other parents mastered 19 protocols. One parent did not master any protocols and left the study at the end of the 2 nd month. The average number of new protocols mastered by parents was 8 (range $0-23$ ) in the first 2 months and 2 (range 0-7) in the last 2 months.

\section{Skills Acquired by PBST Students}

A comparison of the number of skills the PBST students acquired in 2 months before the lockdown (mid-January 2020 to mid-March 2020) with three successive blocks of 2 months each once the intervention commenced is in Fig. 12. The mean number of mastered skills in the in-clinic phase for this cohort of 10 students was 6 (range $0-19$ ), and it increased to 23 (range 4-49) by the end of the study. In the first block of 2
Fig. 2 Equipment Used at Parents' End and Behavior Supervisors' End for Parent Behavioral Skills Training Sessions

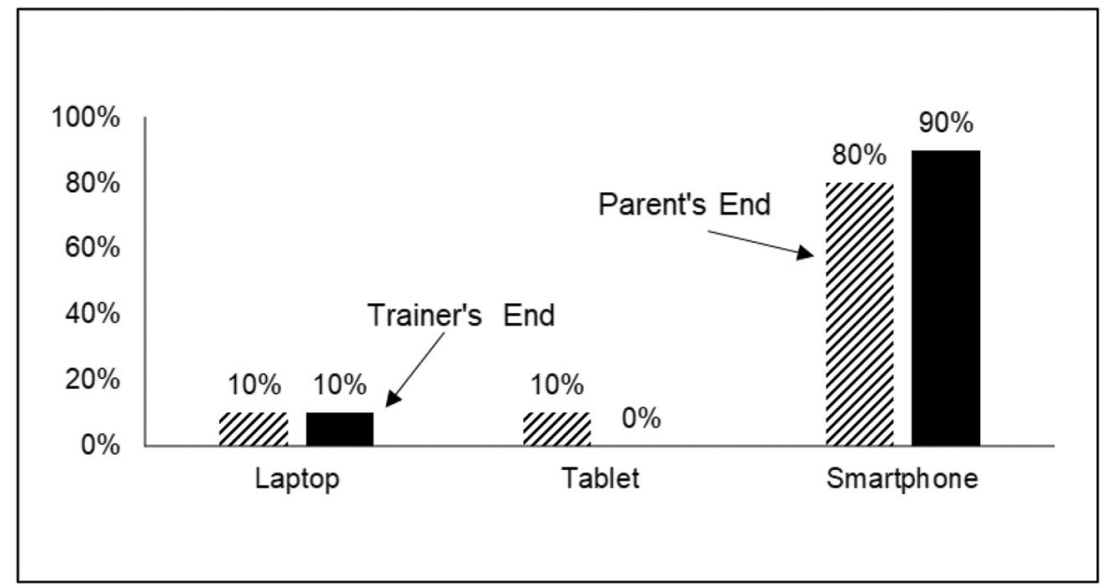


Fig. 3 Number of Students From the Cohort Undergoing Intervention

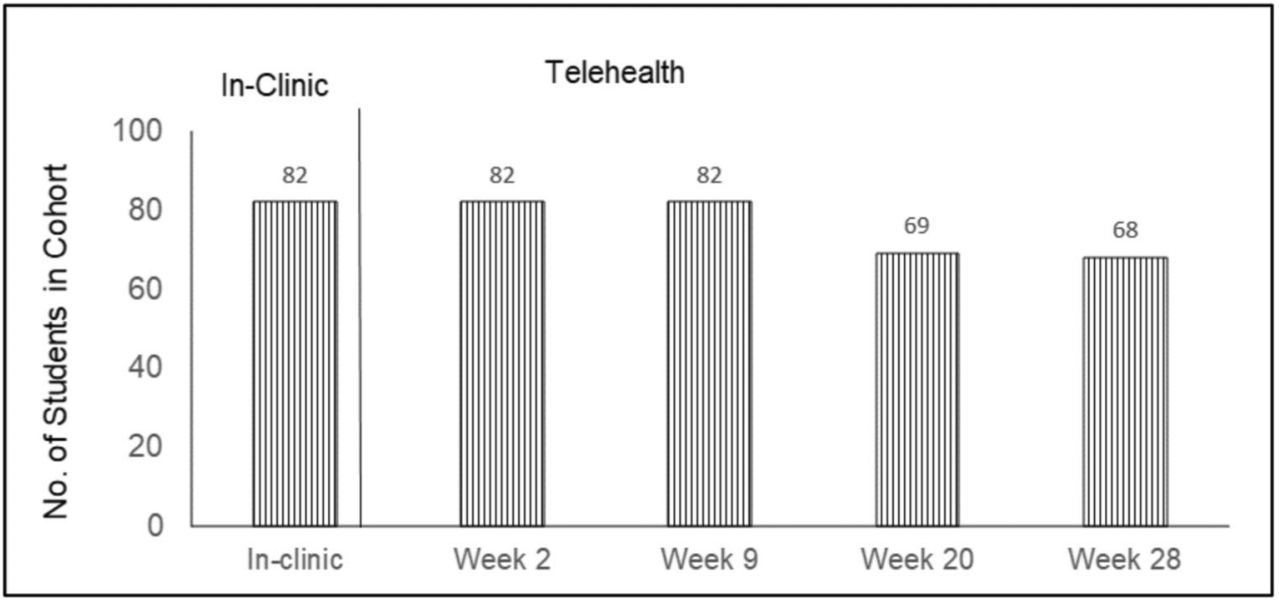

months, 6 out of 10 students acquired more skills in the telehealth condition than the in-clinic condition. From the 3rd month, all five students who had continued acquired a greater number of skills than in the in-clinic condition.

\section{Logistical and Ethical Challenges}

The qualitative research, conducted using in-depth interviews with the clinical director and the behavior supervisors, uncovered several challenging decisions, implementation challenges, and barriers to telehealth sessions' smooth conduct. A summary of the findings from the interviews and the factors that influenced the decisions in the areas of initial enrollment decisions, adherence to ethical standards, and operational decisions are in Table 6.

\section{Social Validity}

The results of the parent survey are in Table 2. Of the 32 parents who filled out the online form and submitted it, though
$66 \%$ of the parents felt that their child had acquired fewer skills in the lockdown, more than $80 \%$ rated the telehealth initiative, therapist's enthusiasm, and clinical recommendations as excellent or very satisfactory. Though unconventional, as in the Espinosa et al. (2020) study, parent responses to an open-ended question are presented in the Appendix.

\section{Discussion}

All $75 \%$ of the students who stayed on in the study continued to acquire new skills, though the mean session durations were $30 \%$ of the in-clinic hours over the 6-month telehealth intervention for TSD and TSPM students. This continued acquisition suggests that telehealth implementation holds promise for the continuation of services in lockdown conditions for a significant proportion of the students whose parents can commit even a limited number of hours daily. The distance support model outlined in Espinosa et al. (2020)
Fig. 4 Therapist-Student ParentMediated (TSPM) Students Who Left the Program and the Reasons

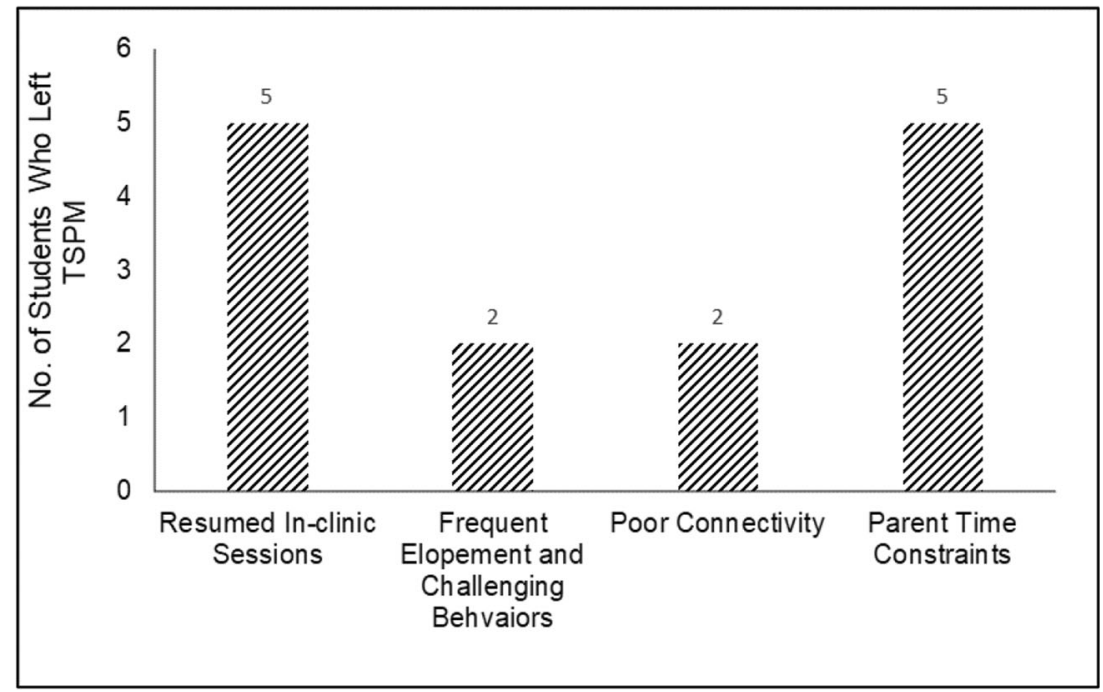


Fig. 5 Session Durations for Therapist-Student Direct and Therapist-Student ParentMediated Students in Minutes and as a Percentage of In-Clinic Hours

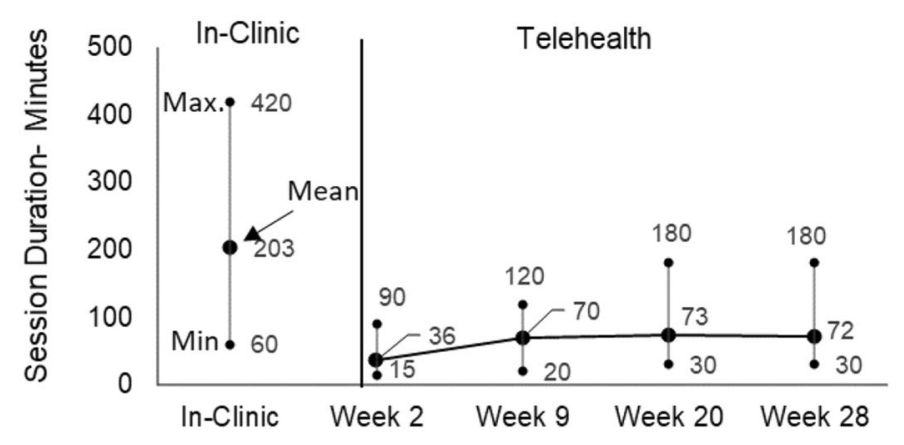

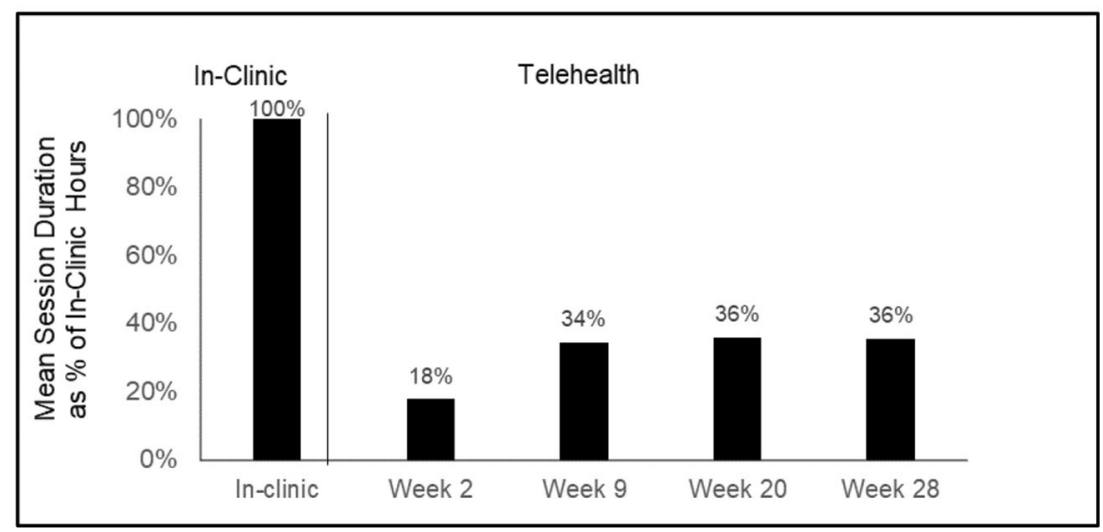

involved using only electronic materials for instruction. Therapists in this study used PowerPoint slides or electronic materials on laptops only with $19.5 \%$ of the TSD and TSPM student sessions and $10 \%$ of the PBST parent sessions. In countries like India, laptops may not be available to all therapists and students. This study confirms that ABA-based skill acquisition interventions can be provided with smartphones over the internet, with materials available at therapists' and students' homes.
In the in-clinic sessions, on average, parents needed to commit only $45 \mathrm{~min}$ to $1 \mathrm{hr}$ every month for in situ observations and an additional hour every alternate month for case meetings. However, telehealth required parents' presence daily to facilitate the sessions, especially for the TSPM students. Perhaps because of this requirement and reduced family incomes, the mean session duration (Fig. 6) was much lower during the telehealth phase than the prelockdown, in-clinic phase. However, once the telehealth interventions
Fig. 6 Parent Behavioral Skills Training Session Durations in Minutes per Week

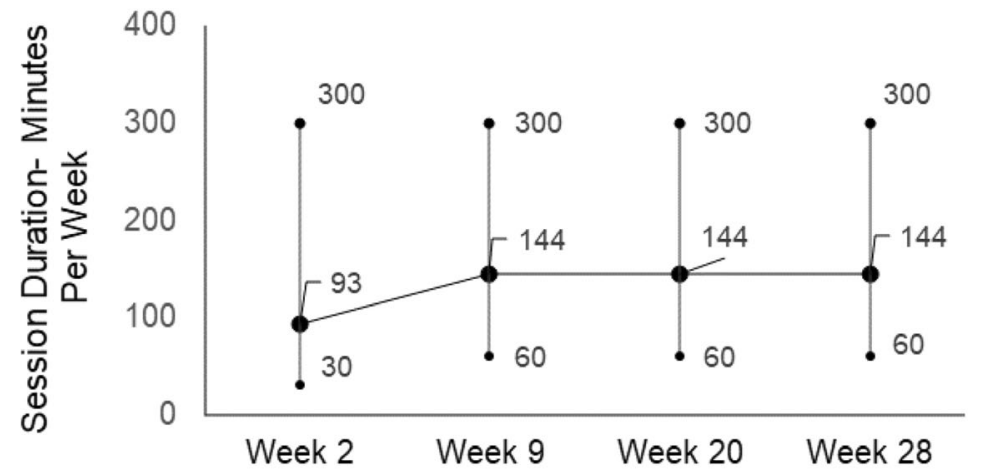


Fig. 7 Instructions per Hour for Therapist-Student Direct and Therapist-Student ParentMediated Students

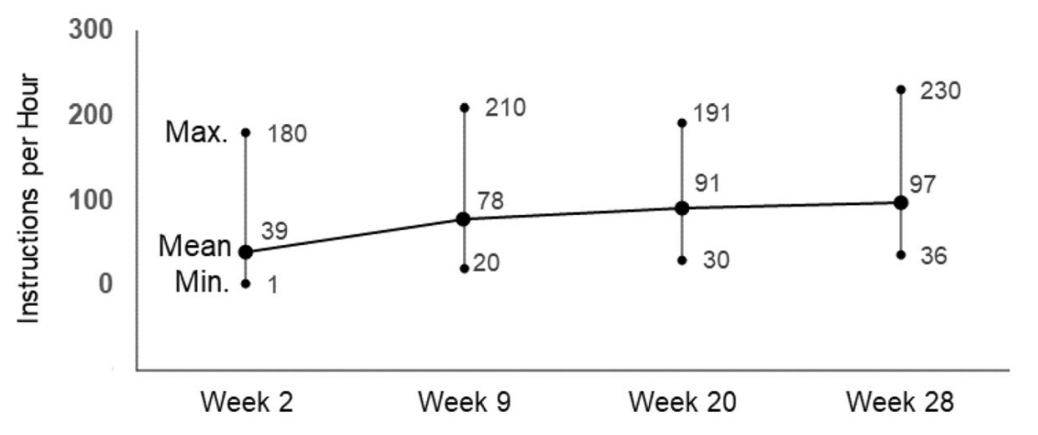

commenced, the mean session duration still increased by $69 \%$ between the first 2 months and the last 2 months.

In addition to session durations, the number of instructions per hour and the number of skills mastered by the students can be useful indicators of the telehealth service delivery's maintainability and sustainability. The mean number of trials conducted per session for TSD and TSPM students doubled between the 2 nd week of telehealth services and the 9th and maintained after that. Additional evidence to support the telehealth program's effectiveness for many students is available from the results on students' skill acquisition. All 92 TSD and TSPM students and 9 out of 10 PBST students continued to acquire new skills. Of the TSD and TSPM students, $46 \%$ showed an increase in the number of skills acquired in the initial 2 months of telehealth compared to the in-clinic phase, and this improved to $52 \%$ in the last 2 months. Nearly $50 \%$ of the students acquired fewer skills in telehealth condition compared to in clinic. It is important to analyze if reduced session durations could account for the fewer skills acquired in telehealth.If session durations alone were responsible, then the hours of instruction per mastered skill should remain constant. A comparison of hours of instruction per mastered skill across the two groups of students, one acquiring fewer skills and the other more in the last 2 months compared to in-clinic conditions, is shown in
Figs. 10 and 11. These results suggest that there may also be student characteristics that could account for the difference. The students who acquired fewer skills in the last 2 months compared to the in-clinic condition required more hours of instruction per skill across the 6 months (Fig. 10).

However, students who acquired more skills in the last 2 months required nearly the same number of instruction hours per skill acquired up to the 4th month and required much fewer hours, $3.0 \mathrm{hr}$ versus $4.5 \mathrm{hr}$ in-clinic, at the end of the study. This difference suggests that a reduction in session duration alone may not explain why nearly $50 \%$ of the students acquired fewer skills. Additional factors such as participant characteristics, the degree of presence of prerequisite skills, and the type of skills acquired may need to be explored in future studies to better interpret the learning outcomes in telehealth sessions.

The current study extends previous studies on the use of telehealth to train parents to implement ABA-based interventions. Of the 10 parents implementing interventions with their student-child, 9 were able to implement ABA-based protocols with fidelity with their students-childin the first 2 months. In these 6 months, 2 of the 10 parents could continue and train their students/children on the full range of skill acquisition targets (15 or more skills) from in-clinic sessions before the lockdown. The remaining eight parents learned to implement
Fig. 8 Mean Mastered Skills per Student for Therapist-Student Direct and Therapist-Student Parent-Mediated Students

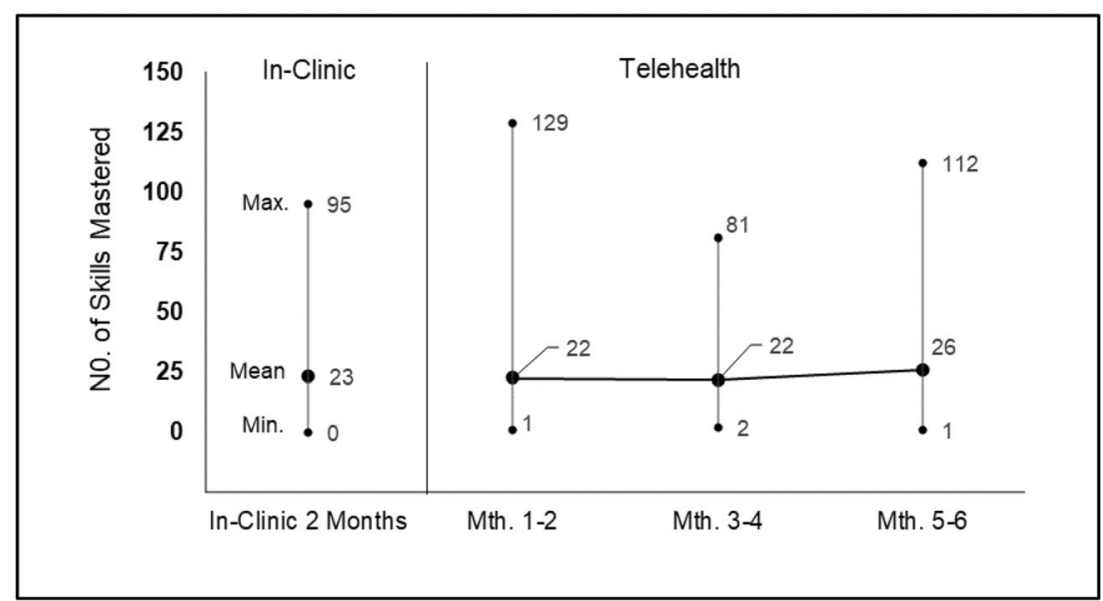


Fig. 9 Scatterplot Depicting the Number of Skills Acquired In Clinic and in Last 2 Months for 68 Therapist-Student Direct (TSD) and Therapist-Student ParentMediated (TSPM) Students. Note. Skills acquired by 68 TSD and TSPM students in 2 months in clinic and 2 months at the end of the study. Each student's data are represented by a dot. Multiple students' data that are identical were plotted as a single dot.

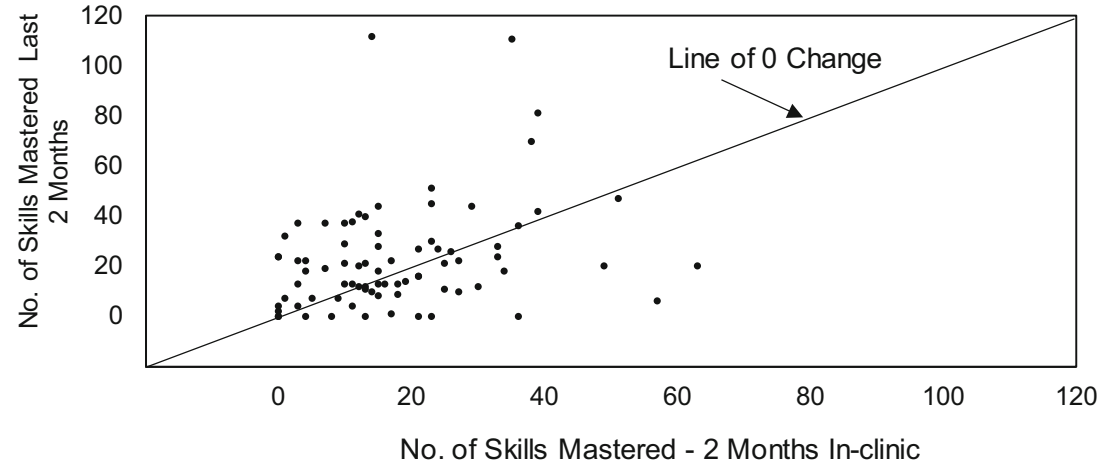

between 5 and 10 protocols. In the subsequent blocks of 2 months, though the number of new protocols mastered by them was fewer, their children continued to master new skills within the domains trained. The PBST sessions in later months were less focused on training the parent on new protocols. The emphasis changed to implementation, providing feedback, problem solving, and facilitating continuous improvement in implementation. The children of parents who continued with the PBST sessions continued to acquire more than 20 skills in 2-month blocks, comparable to the average number of skills the students acquired in the TSD and TSPM sessions. The mean number of skills acquired by PBST students was low in the in-clinic phase before the lockdown. The low average in the in-clinic condition was because one student joined only 10 days before the lockdown, and two other students attended less than $25 \%$ of the in-clinic sessions. This study used presession instructions, role-plays, practice,

Table 5 Number of Students for Whom the Number of Skills Acquired Increased or Decreased Compared to In Clinic

\begin{tabular}{|c|c|c|c|c|c|c|}
\hline \multirow[t]{2}{*}{ Mode } & \multicolumn{2}{|c|}{ Months $1 \& 2$} & \multicolumn{2}{|c|}{ Months $3 \& 4$} & \multicolumn{2}{|c|}{ Months $5 \& 6$} \\
\hline & No. & $\%$ & No. & $\%$ & No. & $\%$ \\
\hline \multicolumn{7}{|l|}{ TSD and TSPM } \\
\hline Total students & 82 & & 69 & & 68 & \\
\hline Increased & 38 & 46 & 33 & 48 & 35 & 52 \\
\hline Remained equal & 1 & 1 & 0 & 0 & 2 & 3 \\
\hline Decreased & 43 & 52 & 36 & 52 & 31 & 45 \\
\hline \multicolumn{7}{|l|}{ BST } \\
\hline Total students & 10 & & 5 & & 5 & \\
\hline Increased & 6 & 60 & 5 & 100 & 5 & 100 \\
\hline Remained equal & 0 & 0 & 0 & 0 & 0 & 0 \\
\hline Decreased & 4 & 40 & 0 & 0 & 0 & 0 \\
\hline
\end{tabular}

Note. $\mathrm{TSD}=$ therapist-student direct; TSPM $=$ therapist-student parent mediated; BST $=$ behavioral skills training. feedback, and question-and-answer sessions as BST components. The results suggest that the BST treatment package composed of these components was useful.

The social validity results compiled from 32 parent responses to a survey were mostly positive. They suggest that parents welcomed continuing ABA-based intervention services through telehealth. The high ratings on the telehealth initiative and therapist efforts and enthusiasm and an increase in parent familiarity with and confidence in ABA science lend strength to telehealth's social validity. However, $66 \%$ of the parents reported that their children acquired fewer skills in telehealth sessions than in clinic. BMI will need to address this perception by addressing factors such as student prerequisites and adaptation of the skills targeted. BMI could also address fading parents' presence, which could help increase session durations.

There were several transition-related challenges and decisions. One of the significant challenges was that the process depended on the availability of smartphones and sufficiently high bandwidth at the students' and the therapists' ends. Though living in metropolitan cities, some families could not ensure good connectivity in their homes. Another challenge was determining the ideal videoconferencing platform, given the lack of rigorous and credible guidelines on data privacy and safety in the country. Due to parents' familiarity and preference, therapists and behavior supervisors used Skype with Meet Now and WhatsApp video calling for telehealth sessions. However, it may become necessary to migrate instructional sessions to other platforms recommended for greater safety and security standards. It would further require sensitization of the parents and the training of therapists in the use of such platforms. The implementation of telehealth service delivery necessitated securing additional informed consent agreements from parents. The first was consent for telehealth, and the second for recording telehealth sessions for training and quality purposes. In a clinic 
Fig. 10 Instruction Hours per Skill for Therapist-Student Direct (TSD) and Therapist-Student Parent-Mediated (TSPM)

Students Who Acquired Fewer Skills. Note. This figure presents mean instruction hours, per student, per skill acquired by TSD and TSPM students who acquired fewer skills in the last 2 months of the intervention compared to 2 months in clinic.

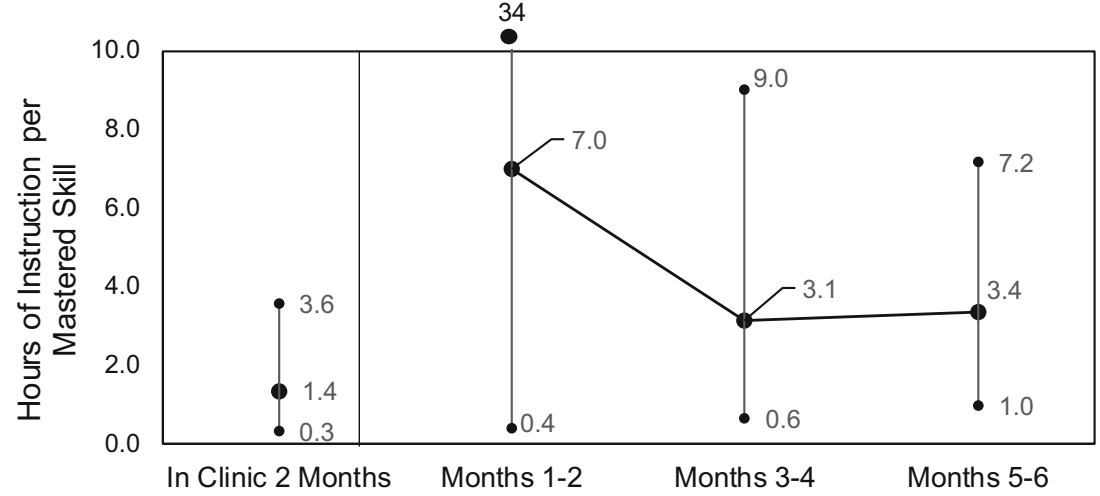

setting,BMI could implement several safeguards for privacy and data security. All therapists had ethical conduct guidelines incorporated into their employment contracts. Also, therapists would deposit their mobile phones with an administrator and access them only during scheduled breaks. This arrangement was to ensure that sessions with students do not get recorded inadvertently. In a telehealth service delivery system,BMI had to introduce safeguards to ensure that personnel did not record sessions without additional permissions. Even with students for whom informed consent was available, the behavior supervisor or the clinical director instructed therapists by email to record specific sessions. Once they completed a session recording, they uploaded the recording to a dedicated secure site and deleted the videos from their phones.

There were additional ethical and operational challenges that needed careful consideration and decision making (Table 6). Some challenges were operational and became significant as the implementation progressed. The first involved sustaining parent motivation and availability to facilitate sessions. In-clinic sessions had the advantage of very structured entry and exit times. One of the telehealth implementation challenges was that some parents requested ad hoc postponements or frequent changes to scheduled times depending on how they organized their days. These changes caused inconvenience to therapists and behavior supervisors who had to work hard to rearrange their schedules. When parents were out of the frame in some sessions, it was not clear whether the students were receiving inadvertent prompts. Some of the students would look toward their parent before responding to the therapist in the camera. The therapists addressed this problem by requesting the parent to be seated behind the student during sessions and come forward only for trials where parent prompts were required. In a clinic, a therapist would have stimulus materials standardized and well organized. Because the lockdown was sudden, therapists sourced stimuli from the internet and their homes. In many sessions, especially in the initial months, the stimulus materials were handwritten or hand drawn. As the sessions progressed, behavior supervisors examined the stimuli used closely and suggested replacements and improvements. With TSD students, where the student had a laptop and the therapist did not, the therapist emailed requests for specific stimulus materials, such as worksheets, ahead of the session to the parent. These had to be printed or handwritten by parents at home. In such sessions, the stimulus materials would often not be available on time, requiring additional follow-up with parents. In some telehealth sessions, parents required additional training to avoid re-presenting the discriminative stimuli or prompting without the therapist's signal.
Fig. 11 Instruction Hours per Skill for Therapist-Student Direct (TSD) and Therapist-Student Parent-Mediated (TSPM) Students Who Acquired More Skills in the Last 2 Months. Note. This figure presents instruction hours per skill acquired by TSD and TSPM students who acquired more skills in the last 2 months of the intervention compared to 2 months in clinic.

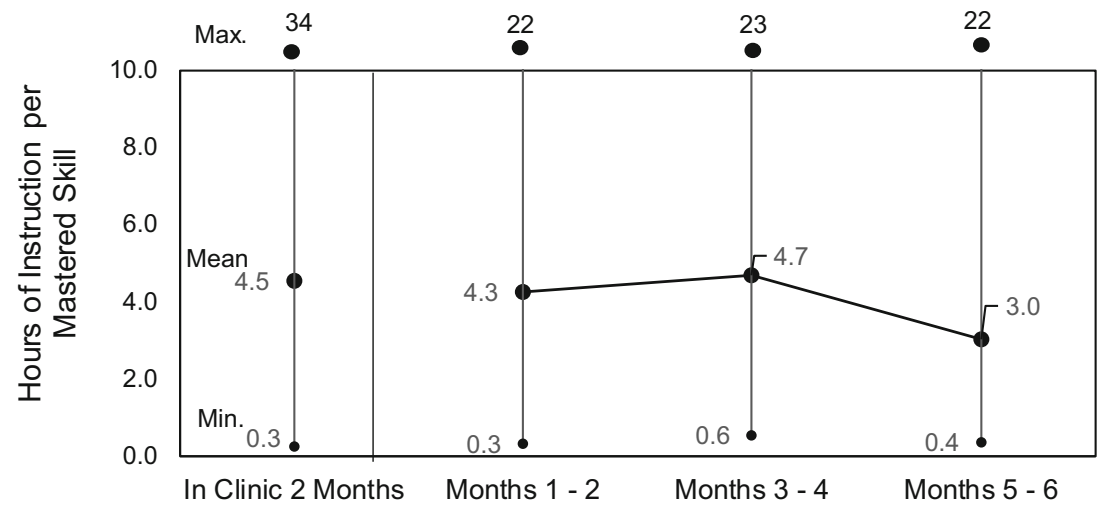


Fig. 12 Mean Number of Protocols Mastered per Parent and Skills Acquired by Their Children

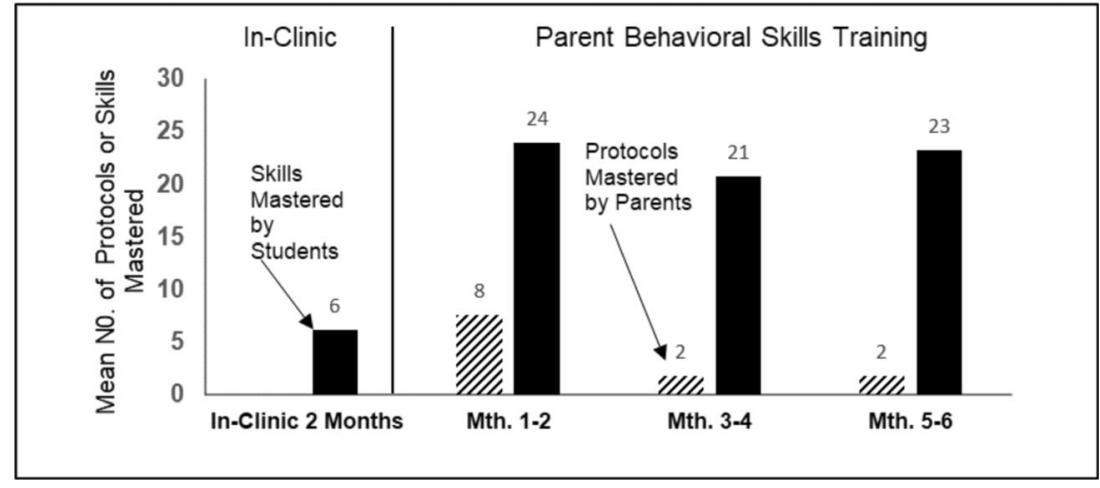

Another ethical issue was recording videoconferences, a functionality available on most video meeting sites. Where an organization provides services through many staff members, it has to have safeguards, both contractual and procedural. In this study, informed consent was first secured from clients' parents, specifically for telehealth as the service delivery model. Also, additional terms were agreed and incorporated into the employment contracts with staff delivering the services. These included uploading session recordings to a designated secure site and deleting them from their phones. However, as a matter of extraordinary caution, service providers could consider having a few dedicated personnel in the organization authorized to host sessions when recordings are required. Given the number of students who successfully continued to acquire skills in this study, the right to continue effective treatment requires practitioners to gain necessary expertise and implement telehealth services where or when inclinic services are not available. The safeguards concerning securing additional informed consents due to the nature of the platforms used need to be planned with due consideration and implemented. National and international bodies that govern ethical conduct in behavior analysis could draw up additional telehealth-use guidelines.

The current study extends Frederick et al.'s (2020) study by addressing a population of students with autism spectrum disorder. It also extends Espinosa et al. (2020) by transitioning the learning targets from the in-clinic intensive behavioral intervention program, such as mands, tacts, intraverbals, imitation, listener responding, reading, and math. Also, it extends previous studies on parent training using BST by using BST over telehealth for training parents to use ABA-based procedures with their student-childr to target language and communication skills from their intensive behavioral intervention program.

The study has certain limitations. Many parents, more than 150 at the beginning of the study, did not opt for the continuation of services using telehealth. Due to a lack of time,BMI did not make efforts to contact them and better inform them of the options. Of the parents who opted in, for different reasons described in this study, 23 out of $92(25 \%)$ opted out of the telehealth program due to poor connectivity, an inability to commit time to facilitate sessions, and the student's noncompliance or escape behaviors in telehealth sessions. Future studies could examine communication, education, and other factors to dispose parents favorably toward telehealth services. The number of instances of increases and decreases in the variables of interest - namely, session durations, number of trials, and acquired skills - was studied. With a significant proportion of the students, there were therapeutic increases in the variables of interest. There were also many students for whom the rate of skill acquisition per block of 2 months during telehealth was lower than in clinic. One of the primary reasons is a substantial reduction in telehealth session durations compared to in-clinic sessions. In an in-clinic setting, a student's education plan would include skills in domains such as mands, cooperation, shopping, play skills, and group skills in addition to language and academic targets. In telehealth sessions, the emphasis tended to be more on domains such as tacting functions, features, and class; grammar rules; and math, especially for TSD students. Although this study compared the numbers, it did not attempt to compare the skills taught and acquired in clinic versus telehealth. Future researchers could rate skills based on their complexity to effectively compare them against skills that could be more easily acquired using telehealth. Future researchers could look at enablers and barriers related to students, families, and therapists and conduct well-controlled studies to examine their effects. BMI could have taken feedback from parents who did not participate in the survey (60 out of 92) over the phone to gain additional information on the telehealth intervention's social validity. Future researchers could also consider surveys conducted by a neutral third-party agency, promising complete anonymity of their responses to minimize bias in survey responses.

This study enumerates the challenges and problems faced during implementation but does not provide quantitative information. For example, the number of times sessions had to be rescheduled or extended to make up for time lost due to connectivity issues is not captured. Also missing is information on the percentage of sessions when TSD students' 
Table 6 Decision Areas and Rationale

\begin{tabular}{lll}
\hline Decision areas & Decision & Considerations \\
\hline $\begin{array}{c}\text { Initial decisions on } \\
\text { enrollment and } \\
\text { service delivery }\end{array}$ & $\begin{array}{c}\text { Participant profile } \\
\text { for a successful } \\
\text { transition }\end{array}$ & $\begin{array}{l}\text { Right to effective treatment } \\
\text { Does the student have any challenging behaviors? } \\
\text { What was the student's attendance span in in-clinic ses- } \\
\text { sions? How does the student score on parameters of co- } \\
\text { operation? }\end{array}$ \\
& $\begin{array}{c}\text { Which therapists } \\
\text { can be involved } \\
\text { in the transition }\end{array}$ & $\begin{array}{c}\text { Is the therapist recently recruited and under training? Can } \\
\text { the therapist commit to a certain number of hours daily to } \\
\text { work from home without distractions? }\end{array}$
\end{tabular}

Ethical considerations

Technology for communications with parents

Informed consent Are the existing informed consent agreements adequate?

Almost all messaging platforms carry a risk of hacking and risks to privacy. Are there any adverse government advisories in India against specific platforms? With what platforms did parents feel at ease?

Technology for There were no regulatory requirements in India like HIPAA video meetings and FERPA in the United States. Is there any advisory against the use of any specific video meeting platforms? With what platforms were parents most comfortable?

Recording sessions

Is there a higher risk of inadvertent video sharing outside the organization?

Can the students attend a telehealth session independently and self-administer reinforcers? Will parents be required for prompting and reinforcer delivery? Can one parent commit to being available throughout every telehealth session?

instruction delivery

\section{Assigning therapists to students}

Was the therapist implementing programs with the student in clinic? Does their availability match with the parents requirements?

Initial targets

The priority is to ensure that the student sits and attends to the camera for the specified initial duration.

Skills to be taught The limitations imposed by mode used

Data collection Cloud-based data collection and collation
Decisions taken

All students whose parents applied, except students with high-rate challenging behaviors, would be accepted. For students with a low attendance span, tasks would be started with a reduced duration and the duration of attendance to tasks would be shaped.

Only therapists who have worked with BMI for at least 6 months and have trained in most of the protocols could conduct telehealth sessions. Therapists who had constraints at home with too many family responsibilities would be exempt. Only therapists who could commit to being available daily for assigned hours would be selected.

WhatsApp messenger would be used, as most parents and therapists were comfortable with its use, and there were no adverse advisories from the government.

Additional informed consent agreements with parents were to be secured by email: the first for telehealth use for instructional sessions and the second for recording meetings for internal training purposes.

There was an advisory note against the use of the Zoom app. Skype "meet now" would be used and technologies and the decisions would be reviewed after a year.

Therapists would be trained to record sessions only on email instruction from a behavior supervisor or clinical director.BMI further would further ensure that they uploaded the recordings to a secure site and deleted them from their equipment by 5:30 p.m. the same evening. Existing agreements with therapists would address adherence to client confidentiality requirements.

TSD: A therapist would run telehealth sessions directly with verbally interactive students who had a record of task-attendance span of $20 \mathrm{~min}$ or more.

TSPM: A therapist would conduct instructional sessions, seeking parent assistance for prompts.

PBST: Behavior supervisors would train parents in implementing ABA-based procedures using telehealth and BST.

As far as possible, the same therapist-student dyads as in the in-clinic sessions would be continued. If such therapist was not available, another therapist who has implemented similar programs as required by the student's IEP would be assigned.

All the students would be started with maintaining mastered skills to ensure cooperation. Targets would be added gradually to their IBI plan. Where required, parents would assist in prompting the student to attend to the camera.

TSD students: Therapists would derive targets from the students' IBI plan, leaving out manding for tangibles. There was no such restriction for TSPM students. BST student targets would be based on their IBI plan but limited based on the protocols that the behavior supervisor trained their parent in.

Therapists would be trained to use Google Sheets on their phones. In some instances, they would send photographs of the data noted on paper, and the administrator would enter the data into Google Sheets.

Note. HIPAA $=$ Health Insurance Portability and Accountability Act; FERPA $=$ Family Educational Rights and Privacy Act; TSD $=$ therapist-student direct; TSPM = therapist-student parent mediated; PBST = parent behavioral skills training; ABA = applied behavior analysis; IEP = individualized education program; IBI = intensive behavioral intervention. 
materials were not ready. Lerman et al. (2020) provide a detailed checklist of more than 15 operational issues that could interfere with implementation and offer solutions to mitigate them. Future researchers could explore data on overcoming such challenges using the solutions suggested and study the effect of such corrective measures on session durations, trials per hour, and mastered skills. Finally, whereas Lindgren et al. (2016) provided calculations to show that telehealth service delivery can be significantly less expensive, this study did not compare the cost of telehealth sessions with the cost of inclinic sessions. Future studies can address this through replications in other geographies and examine whether the lower costs can help extend services to larger populations. In countries like India, consumer awareness about data security problems and privacy issues is low even among educated consumers. As such, it becomes the behavior analyst's responsibility to seek out reliable and safe technologies compliant with international standards for data protection. The review of secure media for communication and videoconferencing was not completed during the study and remains a limitation.
However, the applications used did not have any adverse regulatory restrictions in the country.

In conclusion, despite the limitations outlined, given the uncertain times and the possibility of limited in-clinic attendance, these findings suggest that distance support using telehealth, technology, and parent training is a tool for behavior analysts to deploy, monitor effectiveness, and improve. Due to the low availability of credentialed professionals and the relatively high cost of ABA services, penetration is limited to metropolises in countries like India. However, given the high smartphone penetration, it also offers a possible costeffective option for extending services to rural villages, where $80 \%$ of the population resides and service availability is low. Other countries such as Pakistan, Bangladesh, Singapore, Malaysia, and Nepal, as well as countries in Africa, have very few credentialed behavior analysts and could benefit from a telehealth service delivery model.

\section{Appendix}

Table 7 Some Parent Responses to the Open-Ended Question "Comments on Services Received"

\begin{tabular}{|c|c|}
\hline Parent & Response \\
\hline $\begin{array}{c}\text { Parent } \\
\text { A }\end{array}$ & $\begin{array}{l}\text { Thank you "BMI" for the support during this lockdown period. Our child has improved in his sitting tolerance, attention and other areas. Though Direct } \\
1-1 \text { sessions are better than camera sessions, now parents are learning a lot during this time and the child knows and is slowly adapting to virtual } \\
\text { learning environment. We are now educated on the child's communication and behaviour requirements. The role of ABA is understood clearly and } \\
\text { helps parents to learn and improve the child's each and every activity in a scientific and a target oriented methods. Now we know exactly the role of } \\
\text { parents in the child's development. Customised program from "BMI" for every child is evident and the application of ABA science is sure making } \\
\text { improvements slowly and steadily for our child. "BMI" has the key for any lockdown problems. Thank you to therapists, trainers and management to } \\
\text { ensure consistent support at all the situations. }\end{array}$ \\
\hline $\begin{array}{c}\text { Parent } \\
\text { B }\end{array}$ & $\begin{array}{l}\text { The trainers are so punctual and on time for the virtual classes. Initially there were some tech hitches but we were able to overcome all that in a short span } \\
\text { and were able to move on with your classes. My son used to wander around and didn't understand this concept in the beginning but learnt it over time. } \\
\text { The trainers have been quite effective, with certain constraints, on how to make the hour effective with my son. Their instructions are clear and my son } \\
\text { can follow it. He looks forward to the classes sometimes and is eager. There are only few shortcomings-one of the parent have to be with him all the } \\
\text { time, or else he runs and comes out. Still not able to do it all by himself in the class and the trainer. Since me and my husband are working from home } \\
\text { too, it becomes quite important for us to take time off to sit with him in the class. If we aren't around, he just doesn't sit in the class. }\end{array}$ \\
\hline $\begin{array}{l}\text { Parent } \\
\text { C }\end{array}$ & $\begin{array}{l}\text { "BMI" service awesome, it is helping my kid to progress in his language skills and academics. The approach to make my son to understand the concept is } \\
\text { amazing. Trainers are putting extra efforts, every day they come up with new examples, stories etc.... My son enjoys each session and he will eagerly } \\
\text { wait for next session. Thank you "BMI" HRBR team for all your hard work and support. }\end{array}$ \\
\hline $\begin{array}{l}\text { Parent } \\
\text { D }\end{array}$ & $\begin{array}{l}\text { Excellent! Like I always say "BMI" is the best. I really appreciate the patience of trainers who are training me. "BMI" has given us an opportunity to } \\
\text { understand our kids in more sensible manner. I myself is feeling that confidence in dealing with my son's issue. May Almighty bless the team of } \\
\text { "BMI" with his choicest blessing. }\end{array}$ \\
\hline $\begin{array}{l}\text { Parent } \\
\text { E }\end{array}$ & $\begin{array}{l}\text { There are challenges of online session like child's attention is less, network issues, parents need to be fully involved else session can't take place. Rest } \\
\text { assured I acknowledge the hard work put in by all. }\end{array}$ \\
\hline Parent F & $\begin{array}{l}\text { It was very good. The trainers efforts are highly appreciated. The way they are teaching training is very helpful. Bcoz of the online classes his interactions } \\
\text { with others has improved a lot. Thanks." }\end{array}$ \\
\hline $\begin{array}{l}\text { Parent } \\
\quad \text { G }\end{array}$ & $\begin{array}{l}\text { I am extremely pleased and very appreciative about the services provided by "BMI" during this testing times. All queries are professionally and } \\
\text { assiduously solved to my utmost satisfaction. }\end{array}$ \\
\hline $\begin{array}{c}\text { Parent } \\
\mathrm{H}\end{array}$ & $\begin{array}{l}\text { Excellent initiative by the "BMI" Team. It has been a great help during this period. The trainers, supervisors and clinical director were enthusiastic and } \\
\text { supportive. }\end{array}$ \\
\hline
\end{tabular}


Author Note We thank all the parents, behavior supervisors, and therapists who collaborated in this study, in what must be the most trying time, with the COVID-19 outbreak around the world. The contents of this article are solely our responsibility and do not necessarily represent official views of the organization we are affiliated with.

\section{Declarations}

Ethical approval All procedures performed in this study involving human participants were in accordance with the ethical standards of the institutional and/or national research committee and with the 1964 Helsinki declaration and its later amendments or comparable ethical standards. This article does not contain any studies with animals performed by any of the authors.

Informed consent Informed consent was obtained from the parents of the students who participated in this study.

Conflict of interest The authors declare no potential conflicts of interest with respect to the research, authorship, and/or publication of this article.

\section{References}

Behavior Momentum India. (n.d.). Parents feedback for tele-health. h t t p s://d o c s.g o o g 1 e . c o m/for m s/d/1 l R cwwDfBdT7j5ChrmZgl8eRcvTuHgBhBx0170KsLmg/edit

Carr, E. G., \& Durand, V. M. (1985). Reducing behavior problems through functional communication training. Journal of Applied Behavior Analysis, 18(2), 111-126. https://doi.org/10.1901/jaba.1985.18-111.

COVID-19: Lockdown across India, in line with WHO guidance. (2020, March 27). UN News. https://news.un.org/en/story/2020/03/1060132

Espinosa, F. D., Metko, A., Raimondi, M., Impenna, M., \& Scognamiglio, E. (2020). A model of support for families of children with autism living in the COVID-19 lockdown: Lessons from Italy. Behavior Analysis in Practice, 13(3), 550-558. https://doi.org/10. 1007/s40617-020-00438-7.

Family Educational Rights and Privacy Act of 1974, 20 U.S.C. $§ 1232 \mathrm{~g}$ (1974).

Frederick, J. K., Raabe, G. R., Rogers, V. R., \& Pizzica, J. (2020). Advocacy, collaboration, and intervention: A model of distance special education support services amid COVID-19. Behavior Analysis in Practice. Advance online publication. https://doi.org/10.1007/ s40617-020-00476-1.

Gay, L. R., Mills, G. E., \& Airasian, P. (2011). Educational research: Competencies for analysis and applications (10th ed.). Pearson Education.

Health Insurance Portability and Accountability Act. Pub. L. No. 104191, § 264, 110 Stat. 1936.

Lerman, D. C., O’Brien, M. J., Neely, L., Call, N. A., Tsami, L., Schieltz, K. M., Berg, W. K., Graber, J., Huang, P., Kopelman, T., \& Cooper-Brown, L. J. (2020). Remote coaching of caregivers via telehealth: Challenges and potential solutions. Journal of Behavioral Education, 29(2), 195-221. https://doi. org/10.1007/s10864-020-09378-2.

Lindgren, S., Wacker, D., Suess, A., Schieltz, K., Pelzel, K., Kopelman, T., Lee, J., Romani, P., \& Waldron, D. (2016). Telehealth and autism: Treating challenging behavior at lower cost. Pediatrics, 137(Suppl. 2), S167-S175. https://doi.org/10.1542/peds.2015-2851o.

Microsoft. (2020). What is Meet Now and how do I use it in Skype? Skype Support. https://support.skype.com/en/faq/FA34926/what-is-meetnow-and-how-do-i-use-it-in-skype

Ministry of Home Affairs. (2020). MHA issues advisory on secure use of ZOOM meeting platform. Press Information Bureau, Government of India. https://pib.gov.in/PressReleaseIframePage.aspx?PRID= 1615008
Najdowski, A. C., Chilingaryan, V., Bergstrom, R., Granpeesheh, D., Balasanyan, S., Aguilar, B., Tarbox, J., \& Roane, H. (2009). Comparison of data-collection methods in a behavioral intervention program for children with pervasive developmental disorders: A replication. Journal of Applied Behavior Analysis, 42(4), 827-832. https://doi.org/10.1901/jaba.2009.42-827.

Parsons, M. B., Rollyson, J. H., \& Reid, D. H. (2012). Evidence-Based Staff Training: A Guide for Practitioners. Behavior Analysis in Practice, 5(2), 2-11. https://doi.org/10.1007/BF03391819.

Partington, J. W., \& Mueller, M. M. (2012). The Assessment of Functional Living Skills ${ }^{\mathrm{TM}}$ : An assessment, skills tracking system, and curriculum guide for skills that are essential for independence. Behavior Analysts.

Peterson, S. M., Eldridge, R. R., Rios, D., \& Schenk, Y. A. (2019). Ethical challenges encountered in delivering behavior analytic services through teleconsultation. Behavior Analysis: Research and Practice, 19(2), 190-201. https://doi.org/10.1037/bar0000111.

Pollard, J. S., Karimi, K. A., \& Ficcaglia, M. B. (2017). Ethical considerations in the design and implementation of a telehealth service delivery model. Behavior Analysis: Research and Practice, 17(4), 298-311. https://doi.org/10.1037/bar0000053.

Quigley, S. P., Blevins, P. R., Cox, D. J., \& Brodhead, M. T. (2019). An evaluation of explicit ethical statements in telehealth research with individuals with autism spectrum disorder. Behavior Analysis: Research and Practice, 19(2), 123-135. https://doi.org/10.1037/bar0000094.

Sundberg, M. L. (2008). VB-MAPP Verbal Behavior Milestones Assessment and Placement Program: A language and social skills assessment program for children with autism or other developmental disabilities : Guide. AVB Press, Concord, CA.

Tomlinson, S. R., Gore, N., \& McGill, P. (2018). Training individuals to implement applied behavior analytic procedures via telehealth: A systematic review of the literature. Journal of Behavioral Education, 27(2), 172-222. https://doi.org/10.1007/s10864-018-9292-0.

Tsami, L., Lerman, D., \& Toper-Korkmaz, O. (2019). Effectiveness and acceptability of parent training via telehealth among families around the world. Journal of Applied Behavior Analysis. https://doi.org/10. 1002/jaba.645.

Unholz-Bowden, E., McComas, J. J., McMaster, K. L., Girtler, S. N., Kolb, R. L., \& Shipchandler, A. (2020). Caregiver training via telehealth on behavioral procedures: A systematic review. Journal of Behavioral Education, 29(2), 246-281. https://doi.org/10.1007/ s10864-020-09381-7.

Vaishnav, A. (2019). The Personal Data Protection Bill, 2019: All you need to know. PRSIndia. https://www.prsindia.org/theprsblog/ personal-data-protection-bill-2019-all-you-need-know

Wacker, D. P., Lee, J. F., Padilla Dalmau, Y. C., Kopelman, T. G., Lindgren, S. D., Kuhle, J., Pelzel, K. E., Dyson, S., Schieltz, K. M., \& Waldron, D. B. (2012). Conducting functional communication training via telehealth to reduce the problem behavior of young children with autism. Journal of Developmental and Physical Disabilities, 25(1), 35-48. https://doi.org/10.1007/s10882-012-9314-0.

Wacker, D. P., Lee, J. F., Padilla Dalmau, Y. C., Kopelman, T. G., Lindgren, S. D., Kuhle, J., Pelzel, K. E., \& Waldron, D. B. (2013). Conducting functional analyses of problem behavior via telehealth. Journal of Applied Behavior Analysis, 46(1), 31-46. https://doi.org/ 10.1002/jaba.29.

WhatsApp LLC. (n.d.). About WhatsApp. https://www.whatsapp.com/ about/

World Health Organization. (2020). WHO coronavirus (COVID-19) dashboard. Retrieved November 27, 2020, from https://covid19. who.int/

Publisher's Note Springer Nature remains neutral with regard to jurisdictional claims in published maps and institutional affiliations. 